\title{
Educational intervention to increase parental knowledge and acceptance of pediatric imaging
}

\begin{abstract}
Background: In pediatric healthcare, although properly justified and optimized imaging is often required to support accurate diagnosis, parents may not allow their children to be subjected to radiation imaging due to a perceived health risk. Such denials of service may lead to inaccurate diagnoses and thus compromise the health of children.
\end{abstract}

Aim: The goal of our study was to assess if communicating benefits and risks of pediatric radiation imaging with parents affects their level of comfort and acceptance of imaging.

Methods: We utilized data from a recently released World Health Organization (WHO) publication to construct educational materials (printed pamphlet and phone application) that present basic facts, risks and benefits of radiation imaging to parents. The risk was quantified scientifically in terms of milliSieverts $(\mathrm{mSv})$ of exposure per imaging methods and equivalent days of exposure to the sun. Parents were interviewed before and after the educational intervention.

Results: Following the intervention the level of knowledge and comfort was significantly higher $(p<0.001)$. Parents reported that the educational materials were helpful particularly in lowering their concerns towards radiation imaging.

Conclusion: Our results indicate that communicating relevant information to parents increases their comfort and acceptance of radiation imaging in the pediatric dental practice.
Volume 8 Issue I - 2018

\author{
Jason Zavras,' Haejin Hwang, ${ }^{2}$ Grant \\ Murray, ${ }^{2}$ Abeer Zletni, ${ }^{2}$ Jayapriyaa \\ Shanmugham ${ }^{2}$
}

'Dover Sherborn High School, Dover, Massachusetts, USA ${ }^{2}$ Department of Pediatric Dentistry, Henry M. Goldman School of Dental Medicine, Boston University, USA

\begin{abstract}
Correspondence: Jayapriyaa R. Shanmugham, BDs, MPH, DrPH, Department of Pediatric Dentistry Boston University Henry M. Goldman School of Dental Medicine, 100 East Newton Street, 7th floor, Suite 706 Boston, MA. 02118, USA, Tel 617-638-5038, Fax 617-638-5071, Email jshanmug@bu.edu
\end{abstract}

Received: December 0I, 2017 | Published: January 02, 2018

Keywords: Children, Pediatric dentists, Dental education, Patient communication, Radiology, Imaging risks

Abbreviations: WHO, World Health Organization; $\mathrm{mSv}-$ Milli-Sievert, unit of measurement for effective radiation dose; CT, Computed Tomography; CBCT, Cone Beam Computed Tomography; AAPD, American Association of Pediatric Dentistry; ALARA, As low as reasonably schievable; BUMC, Boston University Medical Center; MIR, Medical Imaging Risk

\section{Introduction}

Few exposures generate more anxiety to the public than exposure to any type of radiation. Images and stories of affected individuals that agonize due to extreme exposure to ionizing radiation (usually during nuclear facility accidents) have captured the imagination of people since the atomic bomb explosions in Hiroshima and Nagasaki during World War II, and have created deep seated fears. ${ }^{1,2}$ Over the years the fear of radiation from the mounting use of medical imaging and from reports in the media that dramatize radiological mishaps may have resulted in patients' hesitation to undergo even the required diagnostic imaging procedures. ${ }^{3}$ Currently there is increased awareness among the medical professionals on the importance of effectively communicating radiation risks to the patients so as to help them make informed decisions.

Effective dose of radiation exposure is expressed in Sieverts (Sv) based on System International nomenclature and the dose emitted in diagnostic imaging is expressed as milliSieverts (mSv). ${ }^{4}$ Radiation exposure from natural sources such as sun rays and radon gas is a constant part of everyday life. Globally, the average radiation exposure from all sources is approximately $3 \mathrm{mSv} /$ year per person, however natural radiation levels vary by geographic locations. ${ }^{5}$ In United States, the average annual radiation exposure to naturally occurring radioactive substances accounts for half of the average annual human exposure, which has been quantified at 3 milliSieverts $(\mathrm{mSv})$. The other half is due to artificial sources of radiation, mainly medical radiography. ${ }^{6}$

Medical radiography comes in many forms. While ionizing medical radiation at high doses may cause reversible or irreversible health effects, exposure to controlled, measured, optimized low levels of dental radiography have negligible adverse effects. ${ }^{7}$ New digital and three dimensional radiation imaging methods gain popularity as they allow for advanced diagnostic capabilities that solve the many limitations of two-dimensional conventional radiography. While the capability increases considerably, new imaging methods tend to be associated with higher exposure levels. Computed Tomography (CT) has been the most commonly used diagnostic medical imaging procedure globally and in the United States, emitting higher doses of radiation when compared to other medical imaging techniques such as interventional fluoroscopy and conventional radiography. ${ }^{8}$ Globally, CT represents $6.3 \%$ of all $\mathrm{x}$-ray examinations and contributes to $43 \%$ of the global collective dose. ${ }^{5}$ A CT of the head in a 10 -year old is associated with $2.2 \mathrm{mSv}$ of exposure whereas radiation from dental imaging is relatively low where an intraoral x-ray emits $0.005 \mathrm{mSv}$. The introduction of Cone Beam Computed Tomography $(\mathrm{CBCT})$ in dentistry has generated significant excitement within the clinical community due to the reduced exposure levels as compared with the CT scan, and has enthusiastically been adopted. However many critics raise concerns. The concerns primarily have to do with the possibility of over-exposure to higher levels of radiation, especially for children. For comparison purposes, a posterior bitewing radiography is associated with $0.005 \mathrm{mSv}$ of exposure; a CBCT of the head with significantly less than $1 \mathrm{mSv}^{7}$ While radiation doses are comparatively lower in dentistry, the policy statement on patient 
safety by the American Association for Pediatric Dentistry (AAPD) highlights the importance of using the ALARA principle (As Low As Reasonably Achievable) in dental radiography to achieve a balance between benefits and potential risks. ${ }^{9,10}$

Key stakeholders have differing views about the rapidly expanding use of new technologies, the vast medical benefits of the new methods but also about the risk of increased exposure due to unjustified or inappropriate use of these technologies. Differing views often cause confusion to the patient community. Recognizing the need for increased dialogue between the clinical community and the patient community, and in an attempt to develop communication tools that facilitate the dialogue, the World Health Organization (WHO) convened an International Workshop on Radiation Risk Communication in Pediatric Imaging in September of 2010, with broad participation. Subsequently a working group was formed that established, pilot tested and ultimately finalized the publication "Communicating Radiation Risks in Paediatric Imaging"?

Research is limited on the knowledge and perception of radiation imaging among parents or guardians of children. In evaluating psychological aspects of communicating radiation risk to patients it is important to understand that the lack of knowledge on radiation dose and level of risk associated with the radiation exposure may be the cause for increased fear among patients. ${ }^{3}$ To help mitigate the fear interactive communications on the benefits versus risks in clear understandable verbiage is vital. A survey among 602 participants in Spain on evaluating effective methods of communicating radiation risk among patients reported that participants preferred oral and written forms of communication with information on radiation doses from medical imaging versus natural radiation. ${ }^{11}$ Another study on evaluating perceptions on radiation exposure among patients, medical students and practicing physicians at a tertiary care community hospital reported that there was lack of knowledge and awareness on the level of radiation exposure not only among patients but also among physicians thus demonstrating the need for further education and training on radiation imaging. ${ }^{12}$ Currently there is limited evidence on the effect of parental education on minimizing anxiety towards radiation imaging for their children. One recent study reported lower anxiety levels among parents and children who received informative material prior to radiology imaging tests when compared to those who did not. ${ }^{13}$ Further research is necessary to evaluate parents' perception on risk communication in the pediatric dental clinic.

Inspired by WHO's report on communicating radiation risks, our goal was to evaluate if communication on risks and benefits has an effect on parental perceptions, comfort level and acceptance of dental recommendations to subject children to necessary dental radiography. The main hypothesis was that a short educational intervention in the dental office will create a significant positive difference in the level of comfort and acceptance of dental x-rays among participating caregivers.

\section{Materials and methods}

\section{Location}

In this pilot interventional study, parents or guardians of children under 18 years of age who were patients at the Pediatric Oral Healthcare Center at the Boston University Goldman School of Dental Medicine (GSDM), Boston, Massachusetts, were included in this study. The study protocol was reviewed and approved by the Boston University Medical Center (BUMC) Institutional Review Board (IRB).

\section{Recruitment and enrollment}

Since our pilot study aims to evaluate the effectiveness of a short educational intervention in a pediatric dental clinic, we recruited a small sample of 50 participants for this initial exploration. Parents or guardians were recruited and interviewed while their children were receiving treatment or consultation in the dental clinic. A simple sampling method was used where parents or guardians of consecutive patients were approached and those who consented were recruited for the study. Only parents or guardians of children under 18 years old and those who were comfortable with the English language were included. Parents of children with serious trauma or in serious discomfort were excluded to avoid putting any patients at risk of delaying their urgent care. The goal of the study was explained to the participants and verbal consent was obtained prior to the interviews.

\section{Measurement and educational tools}

Participants' pre-intervention attitudes and knowledge were assessed with a survey instrument that was developed and pilot tested at GSDM. Information such as parent's race/ethnicity and gender were recorded to capture demographic data to allow for relevant statistical comparisons. Parents were then presented with a written guide describing dental radiography and any questions parents had on the content were addressed by the interviewer. This informational guide used in this study was in English-language and titled "Are Dental Radiographs Safe for Your Child?" The handout was two-sided, colorful and easy to read for parents/guardians, written at the 8th grade level. The handout briefly described the definition and the importance of dental radiography. It also provided a table comparing radiation exposure from several activities with that of background radiation, based on effective whole-body dose. The comparison table included the following sources of radiation and was based on the following dose estimations: annual background radiation with dose estimated at $3 \mathrm{mSv}$; a cross-country flight, at $0.04 \mathrm{mSv}$; an intra-oral radiograph, at $0.005 \mathrm{mSv}$; panoramic, at $0.01 \mathrm{mSv}$; craniofacial cone-beam CT, at $0.2 \mathrm{mSv}$ and chest $\mathrm{x}$-ray, at $0.02 \mathrm{mSv} .8,14$ The handout emphasized the dose associated with routine dental $x$-rays. While the main goal of the handout was to improve parents' knowledge and perception towards dental imaging methods, it also emphasized that the longterm effects of dental radiographs are incompletely understood, and that unnecessary radiation exposure from dental radiographs should be minimized.

A phone app with information on radiation doses and risks was also demonstrated. This app, Medical Imaging Risk (MIR), is designed to inform and educate the patient community about the radiation that various imaging methods elicit (Figures 1\&2). The app is offered free of charge for download by both the Apple and the Android platforms. An interactive discussion took place on the subject of radiation benefits, needs, radiation dose occurring in the natural environment, radiation dose occurring during dental procedures, and possible risks. Parental attitudes towards radiation imaging, understanding, and knowledge were then assessed immediately after the intervention using a postevaluation survey. Only one parent or guardian per treated child was asked to complete the pre- and post-questionnaires. The questions and estimates of radiation exposure and potential future risks are based on information from the relevant literature, and input from two pediatric dentists with survey and content expertise. ${ }^{7,8}$

\section{Pre-questionnaire}

For the pre-questionnaire, parents were asked whether or not any of their children has received a dental radiograph and if they 
Table Continued...

believe that electronic devices such as smartphones produce harmful radiations (allowable answers "yes" or "no"). They were also asked to score their confidence in their knowledge about radiation imaging risks and benefits. In addition, parents were asked their willingness or level of comfort to have their child undergo dental radiographs. For the purpose of this study, we refer "not comfortable" as score 1; "somewhat comfortable" as score 2 ; "very comfortable" as score 3.

\section{Post-questionnaire}

The post-questionnaire included the same questions as the prequestionnaire. In addition, the post-survey evaluated if the educational materials (handout and app) helped in parents' understanding of radiation imaging and if they still had concerns regarding dental radiographs (allowable answers "yes" or "no"). The last question on the post-questionnaire assessed the parents' level of comfort to have their child undergo dental radiographs, scored in the same manner as in the pre-intervention survey.

\section{Data analysis}

Study results were computed, statistically analyzed using STATA version 14.0 statistical analysis software. Descriptive statistics and univariate analysis was conducted to evaluate the effect of the communicating radiation risks through an educational intervention. All statistical tests were two-sided and $p$-values $<0.05$ were deemed statistically significant.

\section{Results and observations}

\section{Results}

During the study period, 54 parents or legal guardians of children treated in the GSDM Pediatric Oral Healthcare Center were asked to participate in the study. Two parents refused and two were excluded due to a significant language barrier during the intervention. A total of 50 parents or guardians participated; 50 pre- and 50 post-intervention questionnaires were included in the analysis. Most of the children in our dental clinic are usually accompanied by their mothers or female guardians. This was reflected in our sample as well as most of the participants were females $(\mathrm{n}=43 ; 86 \%$, Table 1$)$. The sample in this study had a diverse racial and ethnic profile where majority of the participants were African American (40\%) and Hispanic/Latino $(28 \%)$. With regards to insurance type, $82 \%$ of participants indicated that they were enrolled in Medicaid insurance. This distribution reflects the patient population that usually presents at the Boston University Pediatric Oral Health Center.

Table I Characteristics of study participants at the pediatric dental clinic $(\mathrm{N}$ $=50)$

\begin{tabular}{lc}
\hline \multicolumn{2}{l}{ Characteristics of study participants (N = 50) } \\
\hline Gender, $\mathrm{n}(\%)$ & $43(86 \%)$ \\
Females & $7(\mathrm{I} \%)$ \\
Males & \\
Race/Ethnicity, $\mathrm{n}(\%)$ & $\mathrm{II}(22 \%)$ \\
Caucasian & $20(40 \%)$ \\
African American & $14(28 \%)$ \\
Hispanic/Latino & $4(8 \%)$ \\
Asian/Pacific Islander & $\mathrm{I}(2 \%)$ \\
Other & \\
Type of insurance, $\mathrm{n}(\%)$ & $4 \mathrm{I}(82 \%)$ \\
MassHealth*
\end{tabular}

\begin{tabular}{lc}
\hline \multicolumn{2}{l}{ Characteristics of study participants $(\mathbf{N}=\mathbf{5 0})$} \\
\hline Other & $9(18 \%)$ \\
History of previous dental radiographs for participants' child/children, $\mathrm{n}(\%)$ \\
Yes & $39(78 \%)$ \\
No & II $(22 \%)$ \\
\hline
\end{tabular}

*MassHealth is a government health insurance program that serves lowincome families and combines both Medicaid and Children's Health Insurance programs.

Overall among the participants, $78 \%$ reported prior experience with having a child undergoing dental radiograph(s). In the preintervention period, $32 \%$ of parents reported that they have no confidence in their knowledge of radiation imaging benefits, with $46 \%$ having some confidence and only $22 \%$ reporting that they felt very confident (Table 2). These statistics reversed in the post-intervention period, documenting a significant improvement $(p<0.0001)$. The number of participants who reported complete lack of confidence in the pre-intervention survey significantly decreased to $4 \%$ and those who reported complete confidence increased to $56 \%$ following the educational intervention $(p<0.0001)$. A similar trend was observed on the knowledge of radiation risks, again showing a statistically significant improvement $(p<0.0001)$.

With regards to the parents' level of comfort in allowing the use of dental radiographs, $10 \%$ were not comfortable in the preintervention period; $52 \%$ were somewhat comfortable; and $38 \%$ were very comfortable. After the intervention, the percentage of parents who stated that they are not comfortable dropped from $10 \%$ to $2 \%$ while the percentage of the parents who stated they feel very comfortable increased from $38 \%$ to $64 \%(p<0.0001)$. Comparing the pre- and post-intervention periods, the majority $(84 \%)$ of parents or guardians reported that they have no more concerns about pediatric dental radiation imaging following the educational intervention. The majority $(96 \%)$ of the participants reported that they were highly satisfied and found the educational materials to be very helpful. There were no significant differences in the level of comfort on radiation imaging among the different racial/ethnic groups.

\section{Discussion}

The results from our study indicate that there are great benefits in communicating with parents on the topic of pediatric radiation imaging. Providing accurate and authoritative information, such as the one put together by the WHO in their publication "Communicating Radiation Risks in Paediatric Imaging" and easy to use tools that facilitate communication, such as the MIR phone app, seem to empower caregivers and effectively reverse many parents' reluctance to allow their children to go through pediatric dental imaging.?

Recent evidence shows that while the level of awareness among dentists on radiation hazards is at an acceptable level; the communication of their knowledge to the patients is inadequate. ${ }^{15}$ It is vital that health care professionals understand that effective communication includes interactive dialogues with the patients, on the benefits versus risks of the imaging procedures. It is the obligation of healthcare professionals who order or perform radiological imaging, to monitor the scientific literature and to update the data periodically in order to consistently communicate accurate information to patients. In our study a brief educational handout was used to communicate basic information on the benefits and doses of radiation in dental radiation imaging. During the interview, there was an interactive dialogue that improved parents' level of comfort and confidence in the post-intervention survey. 
Table 2 Evaluation of parental knowledge and attitudes of radiation imaging among pediatric caregivers at the GSDM Pediatric Oral Health Center ( $\mathrm{N}=50)$

\begin{tabular}{|c|c|c|c|c|}
\hline \multirow{2}{*}{ Radiation Imaging } & \multicolumn{3}{|c|}{ Pre-test questionnaire Post-test questionnaire } & \multirow[b]{2}{*}{$p$-value } \\
\hline & n (\%) & n (\%) & & \\
\hline \multicolumn{5}{|c|}{ Level of confidence in knowledge about benefits } \\
\hline Not confident & $16(32)$ & $2(4)$ & & \\
\hline Somewhat confident & $23(46)$ & $20(40)$ & & \\
\hline Very confident & II (22) & $28(56)$ & & $<0.0001 *$ \\
\hline \multicolumn{5}{|l|}{ Level of confidence in knowledge about risks } \\
\hline Not confident & $16(32)$ & $2(4)$ & & \\
\hline Somewhat confident & $24(48)$ & $18(36)$ & & \\
\hline $\begin{array}{l}\text { Very confident } \\
\text { Level of comfort in allowing the use of dental }\end{array}$ & $10(20)$ & $30(60)$ & & $<0.0001 *$ \\
\hline $\begin{array}{l}\text { radiographs } \\
\text { Not comfortable }\end{array}$ & $5(10)$ & $\mathrm{I}(2)$ & & \\
\hline Somewhat comfortable & $26(52)$ & $17(34)$ & & \\
\hline Very comfortable & $19(38)$ & $32(64)$ & & $<0.0001 *$ \\
\hline \multicolumn{5}{|l|}{$\begin{array}{l}\text { Reported understanding of radiation from } \\
\text { electronic devices }\end{array}$} \\
\hline Yes & $38(76)$ & $43(86)$ & & \\
\hline No & $12(24)$ & $7(14)$ & & $<0.0001 *$ \\
\hline $\begin{array}{l}\text { Level of comfort in using dental radiographs by } \\
\text { race/ethnicity before intervention }\end{array}$ & Not comfortable & Somewhat comfortable & Very comfortable & $p$-value \\
\hline White & $2(4)$ & $7(6)$ & $2(4)$ & \\
\hline African-American & I (2) & $13(26)$ & $6(12)$ & \\
\hline Hispanic/Latino & $2(4)$ & $4(2)$ & $8(16)$ & \\
\hline Pacific Islander & $0(0)$ & $2(4)$ & $2(4)$ & \\
\hline Other & $0(0)$ & $0(0)$ & $\mathrm{I}(2)$ & $0.25 \dagger$ \\
\hline \multicolumn{5}{|c|}{$\begin{array}{l}\text { Level of comfort in using dental radiographs by } \\
\text { race/ethnicity after intervention }\end{array}$} \\
\hline White & $0(0)$ & $6(12)$ & $5(10)$ & \\
\hline African-American & I (2) & $5(10)$ & 14 (28) & \\
\hline Hispanic/Latino & $0(0)$ & $6(12)$ & $8(16)$ & \\
\hline Pacific Islander & $0(0)$ & $0(0)$ & $4(8)$ & \\
\hline Other & $0(0)$ & $0(0)$ & I (2) & $0.38 t$ \\
\hline \multicolumn{5}{|l|}{$\begin{array}{l}\text { Change from having concerns to not having } \\
\text { concerns after reading the educational materials } \\
\text { (Post-survey questionnaire only) }\end{array}$} \\
\hline Positive Change & & $42(84)$ & & \\
\hline No Change & & $8(16)$ & & \\
\hline \multicolumn{5}{|l|}{$\begin{array}{l}\text { Helpfulness of the materials (handout and app) - } \\
\text { Post-survey questionnaire only }\end{array}$} \\
\hline Yes & & $48(96)$ & & \\
\hline No & & $2(4)$ & & \\
\hline
\end{tabular}

*Results from Pearson Chi-square analysis.

†Based on results from Fisher's Exact analysis.

In pediatric imaging health professionals encounter with the ethical obligation towards proper justification of pediatric imaging. As effective communication with patients and caregivers is increasingly recognized as critical to care, it is important to ensure that all health care professionals have sufficient education and resources to communicate clearly and effectively. ${ }^{7}$ Research has shown that there is widespread underestimation of doses and risks. ${ }^{16,17}$ In pediatric healthcare, the clinical value of radiation imaging for the correct diagnosis and management of injury and illness in children is unquestionable. However, health care professionals that perform radiological imaging must weigh the benefits against potential risks. This is particularly true in the case of routine screening in the absence of clinical signs of disease. While routine dental radiography presents negligible risk, professional societies have established guidelines for their proper use. For example, the American Academy of Pediatric Dentistry (AAPD) clinical guidelines for the prescription of radiographs requires that radiographs are taken only if they will have clinical utility and there is expectation that "the diagnostic yield will affect patient care".18
It also offers a recommended periodicity table based on the child's caries risk level. For young children classified as "high caries risk", a posterior bitewing exam is recommended every 6-12 months whereas for children at low risk the bitewings should be taken every 12-24 months. The guideline also mentions appropriate dose reduction based on the size of the individual. ${ }^{18}$

Health communication is an essential component of health literacy. As health literacy gradually increases, so does the need of patients to receive information from their health care professionals. In the dental environment, communication between dental care providers and patients can improve oral health outcomes. ${ }^{19}$ In a study on parents of children enrolled in Medicaid were highly concerned that dentists do not provide the information needed to ensure their children have good oral health. ${ }^{20}$ Another study on patient communication indicated that $73 \%$ of African Americans reported that communication from their dentist was rare and that they wanted more information about potential future risks of oral cancer examinations. ${ }^{21}$ A study on parental 
knowledge and attitudes towards dental radiography for children reported a lack of knowledge on dental radiographs. ${ }^{22}$ These trends clearly suggest that parents or caregivers are interested in obtaining more information from their healthcare professionals. In 2006, a survey of pediatricians reported a sustained increase in parental questioning of radiation practices and radiation doses since $2001 .{ }^{17}$ This increased interest was confirmed in our study. The great majority of parents (96\%) agreed to participate in the educational intervention and showed an interest to download the mobile MIR application, and to permanently store it in their mobile phones to use as future reference source (Figures 1\&2).

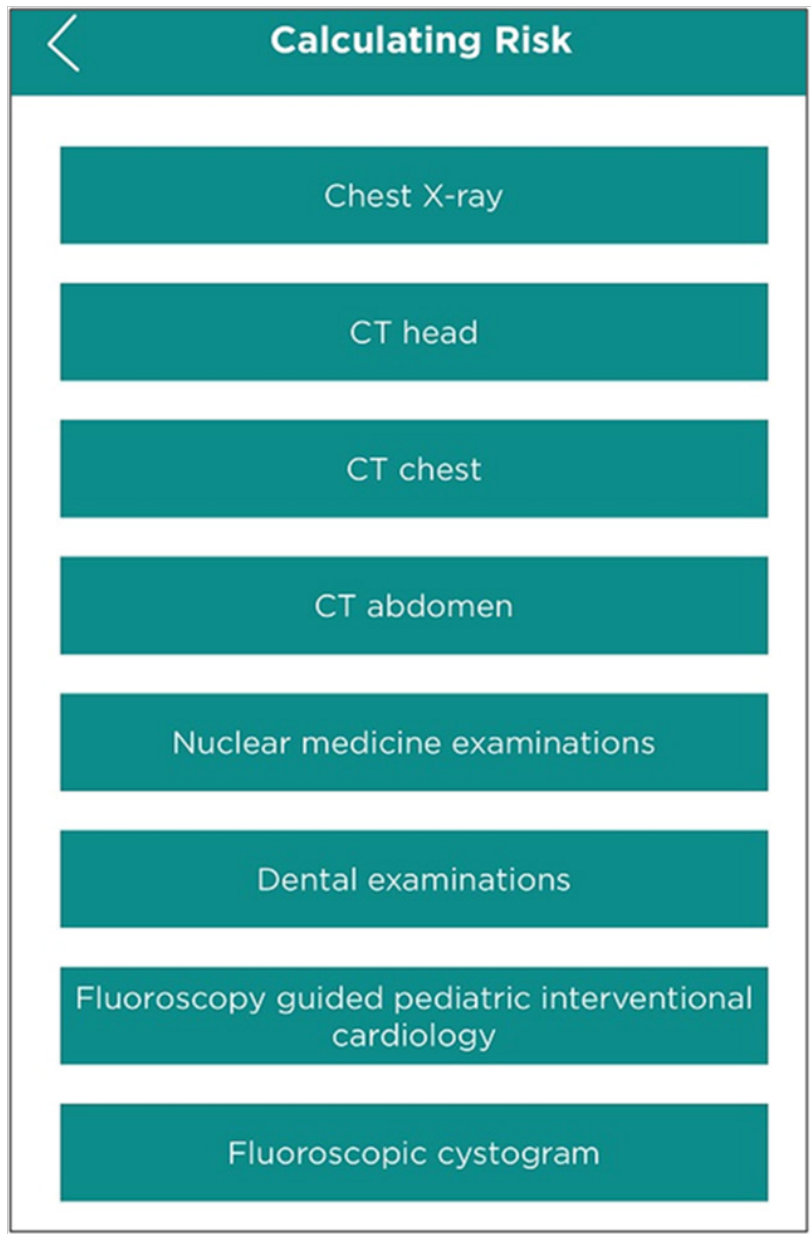

Figure I Medical Imaging Risk (MIR) mobile application: Phone screen image showing types of radiographic tests listed in the application.

Medical and dental radiographs remain the most common artificial source of exposure to ionizing radiation for the U.S population. ${ }^{23}$ Research on patient expectations and current practices for risk communication in medical imaging found that patients want basic information on the level of radiation doses and information on comparison of doses between different sources of radiation. ${ }^{24}$ Providing parents with information on natural background radiation did effectively changed parents' willingness to allow their children to undergo dental radiograph and raised their level of comfort. This finding is consistent with a comparable study by in which approximately $90 \%$ of families preferred to be informed about potential risks before proceeding with medical imaging procedures..$^{25}$ In another study it was shown that when appropriately educated on risks and benefits, parents seem to accept the possibility of slightly increased risk of cancer and to proceed with radiography. ${ }^{26}$

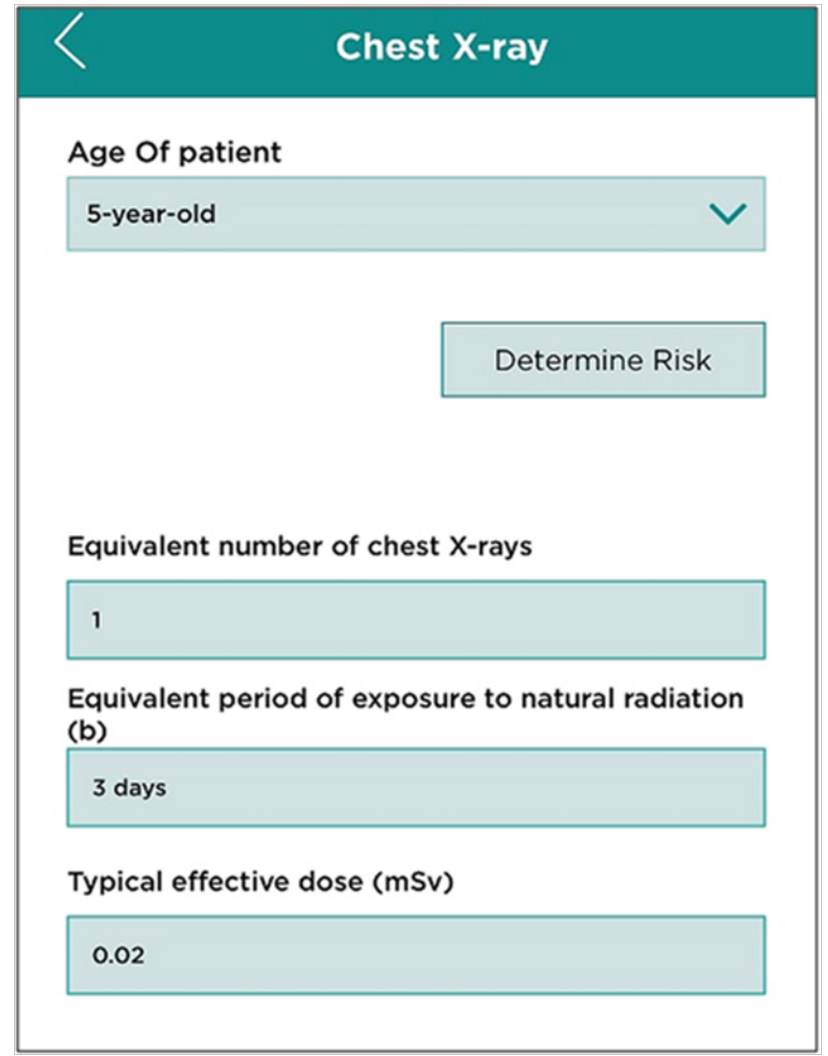

Figure 2 Medical Imaging Risk (MIR) mobile application: Phone screen image showing dose equivalents for selected radiographic test by age.

As health care professionals attempt to better understand the safety issues of medical radiation, particularly in children, the question as to how best to inform parents about this sensitive issue is raised. ${ }^{27}$ Due to the general perception of fear towards radiation imaging, the method of communicating risks is important. ${ }^{3,11}$ Particularly in pediatric healthcare, a major goal of radiation risk communication is to ensure that patients, parents and caregivers receive the information they need in a way that they can make informed decisions. ${ }^{3,28}$ As pediatric imaging involves a broad age range $(0-18$ years $)$, it is critical to consider age-related differences while developing communication strategies. $^{7}$ An important barrier to recognize is that most patients want their own doctors to educate them about ionizing radiation concerns, yet they believe this type of discussion cannot occur due to time pressures in the clinic. ${ }^{24}$ However, in our study we demonstrated that even a short educational session using an informational sheet and an application on the phone is sufficient in improving knowledge and perceptions among parents in the pediatric dental clinic.

Our educational intervention was not time-consuming and can be easily adapted in pediatric dental clinic settings. A previous study reported that parents prefer to receive information through the Internet or through written pamphlets. ${ }^{29}$ In our study, the simple but effective educational intervention tools (printed pamphlet and phone application) that were used reduced or eliminated parental concerns in $84 \%$ of the participants. Change was also evident even among those who categorically do not accept radiographs for their children; the percent was reduced from $10 \%$ initially down to $2 \%$ after the intervention. Based on the results of this study we expect that if such educational interventions are implemented in dental practices, particularly in urban underserved communities that are racially and ethnically diverse, parental acceptance of necessary dental radiographs 
will grow. Limitations of our study include small sample size and lack of gender variability as most of the participants were females which may limit our ability to generalize the study results. The majority of parents or guardians accompanying the children to the dental clinic are females and therefore we were unable to include more male parents or guardians. Another limitation may be the short survey with limited options. However, for ease and convenience to parents who bring their children for treatment we opted to use a short survey. Our goal is to design larger studies with more detailed explorations and analyses.

While it is convenient to use the Internet or social media to communicate to patients in the current digital media era, parents prefer direct patient education and discussion. ${ }^{25}$ In our study, there was direct communication and discussion while the parent reviewed the informational material on radiation imaging. Questions raised by the parents were addressed through an interactive dialogue. Our study results are reflective of past studies reporting a greater level of awareness and comfort after similar interventions. ${ }^{25,26}$

\section{Conclusion}

Effective communication with parents on the topic of risks and benefits of properly justified pediatric imaging leads to increased levels of parental comfort and acceptance. Therefore, it is important to emphasize that the science and art of health literacy and communication on benefits and risks of procedures must be incorporated in the medical and dental curriculum and clinical training programs of professional schools, both at the predoctoral and postdoctoral levels.

\section{Acknowledgements}

None.

\section{Conflict of interest}

The authors in this study declare no conflict of interest.

\section{References}

1. Bromet EJ (2011) Lessons learned from radiation disasters. World Psychiatry 10(2): 83-84.

2. Ohtsuru A, Tanigawa K, Kumagai A, Niwa O, Takamura N, et al. (2015) Nuclear disasters and health: lessons learned, challenges, and proposals. Lancet 386(9992): 489-497.

3. Dauer LT, Thornton RH, Hay JL, Balter R, Williamson MJ, et al. (2011) Fears, feelings, and facts: Interactively communicating benefits and risks of medical radiation with patients. AJR Am J Roentgenol 196(4): 756-761.

4. Brody AS, Frush DP, Huda W, Brent RL (2007) Radiation risk to children from computed tomography. Pediatrics 120(3): 677-682.

5. Report U (2010) Sources and effects of ionizing radiation. United Nations, New York, USA.

6. (2009) Ionizing radiation exposure of the population of the United States. Bethesda, USA.

7. Organization WH (2016) Communicating radiation risks in paediatric imaging: Information to support healthcare discussions about benefit and risk. Geneva.

8. Mettler Jr F, Huda W, Yoshizumi T, Mahesh M (2008) Effective doses in radiology and diagnostic nuclear medicine: a catalog 1. Radiology 248(1): 254-263.

9. Dentistry AAoP (2013) Policy on patient safety.

10. Association AD (2012) The selection of patients for x-ray examination: Dental radiogrpahic examinations. Rockville MD: Food and Drug Administration.
11. Lumbreras B, Vilar J, Gonzalez-Alvarez I, Guilabert M, Pastor-Valero M, et al. (2017) Avoiding fears and promoting shared decision-making: How should physicians inform patients about radiation exposure from imaging tests? PLoS One 12(7).

12. Ricketts M, Baerlocher M, Asch M, Myers A (2013) Perception of radiation exposure and risk among patients, medical students, and referring physicians at a tertiary community hospital. Canadian Association of Radiologists Journal 64(3): 208-212.

13. Johnson A, Steele J, Russell G, Moran R, Fredericks K, et al. (2009) Decreasing pediatric patient anxiety about radiology imaging tests: prospective evaluation of an educational intervention. J Child Health Care 13(4): 370-382.

14. Friedberg W, Copeland K, Duke F, O’Brien III K, Darden EB Jr (2000) Radiation exposure during air travel: guidance provided by the Federal Aviation Administration for air carrier crews. Health Physics 79(5): 591595.

15. Aravind B, Joy E, Kiran M, Shrubin J, Sajesh S, et al. (2016) Attitude and awareness of general dental practitioners toward radiation hazards and safety. Journal of Pharmacy and Bioallied Sciences 8(1): 553-558.

16. Lee C, Haims A, Monico E, Brink J, Forman H (2004) Assessment of patient, physician, and radiologist awareness of radiation dose and possible risks. Radiology 231(2): 393-398.

17. Thomas K, Parnell-Parmley J, Haidar S, Moineddin R, Charkot E, et al. (2006) Assessment of radiation dose awareness among pediatricians. Pediatr Radiol 36(8): 823-832.

18. Dentistry AAoP (2012) Policies and guidelines: Guideline on prescribing dental radiographs for infants, children, adolescents, and persons with special health care needs. Reaffirmed 2012.

19. Yamalik N (2005) Dentist-patient relationship and quality care 3. Communication. International Dental Journal 55(4): 254-256.

20. Mofidi M, Rozier R, King R (2002) Problems with access to dental care for Medicaid-insured children: what caregivers think. American Journal of Public Health 92(1): 53-58.

21. Choi Y, Dodd V, Watson J, Tomar S, Logan H, et al. (2008) Perspectives of African Americans and dentists concerning dentist-patient communication on oral cancer screening. Patient Educ Couns 71(1): 41-51.

22. Chiri R, Awan S, Archibald S, Abbott P (2013) Parental knowledge and attitudes towards dental radiography for children. Aust Dent J 58(2): 163-169.

23. Claus EB, Calvocoressi L, Bondy ML, Schildkraut JM, Wiemels JL, et al. (2012) Dental X-rays and risk of meningioma. Cancer 118(18): 4530-4537.

24. Thornton R, Dauer L, Shuk E, Bylund C, Banerjee S, et al. (2015) Patient perspectives and preferences for communication of medical imaging risks in a cancer care setting. Radiology 275(2): 545-552.

25. Boutis K, Cogollo W, Fischer J, Freedman S, Ben David G, et al. (2013) Parental knowledge of potential cancer risks from exposure to computed tomography. Pediatrics 132(2): 305-311.

26. Larson DB, Rader SB, Forman HP, Fenton LZ (2007) Informing parents about $\mathrm{CT}$ radiation exposure in children: its $\mathrm{OK}$ to tell them. American Journal of Roentgenology 189(2): 271-275.

27. Bulas D, Goske M, Applegate K, Wood B (2009) Image gently: Why we should talk to parents about CT in children. American Journal of Roentgenology 192(5): 1176-1178.

28. McCollough C, Schueler B (2000) Calculation of effective dose. Medical Physics 27(5): 828-837.

29. Hartwig HD, Clingenpeel J, Perkins AM, Rose W, Abdullah-Anyiwo J (2013) Parental knowledge of radiation exposure in medical imaging used in the pediatric emergency department. Pediatric Emergency Care 29(6): 705-709. 